\title{
La Diabetes Mellitus tipo 1 en la escuela. Un estudio narrativo desde la perspectiva familiar
}

Judith Cáceres Iglesias - Universidad de Valladolid

Sara Valdivieso Bermejo - Universidad de Valladolid

Miriam Arribas de Frutos - Universidad de Valladolid

Katherine Gajardo Espinoza - Universidad de Valladolid

Recepción: 25.01.2022 | Aceptado: 31.01.2022

Correspondencia a través de ORCID: Judith Cáceres Iglesias

Citar: Cáceres Iglesias, J, Valdivieso Bermejo, S, Arribas de Frutos, M y Gajardo Espinoza, K (2022). La Diabetes Mellitus tipo 1 en la escuela. Un estudio narrativo desde la perspectiva familiar. REIDOCREA, 11(9), 94-104.

Área o categoría del conocimiento: Ciencias Sociales

Resumen: La diabetes Mellitus tipo 1 es una de las enfermedades crónicas con mayor prevalencia en la infancia, sin embargo, ha sido poco estudiada desde la investigación educativa. El presente trabajo pretende mostrar la realidad escolar del alumnado diagnosticado con esta enfermedad a través del relato de sus familias. Se trata de una investigación de carácter cualitativo, planteada desde la metodología biográficanarrativa. A través del análisis documental, las entrevistas y los relatos de vida se analiza el concepto de educación inclusiva y su aplicación en el día a día de este alumnado. Los resultados reflejan una realidad escolar alejada de la equidad, la cooperación y la justicia, términos imprescindibles a la hora de hablar de educación inclusiva. A partir de los resultados se sugiere reconocer la importancia de la participación familiar en los centros educativos para lograr un correcto control de la enfermedad y fomentar la autoestima de este alumnado. Asimismo, se propone la incorporación de una figura sanitaria en las escuelas que alivie el desgaste emocional, económico y social de las familias. Esta investigación les otorga el protagonismo para que sean sus propios relatos de vida los que muestren sus necesidades e intereses.

Palabra clave: Diabetes Mellitus tipo 1

Type 1 Diabetes Mellitus at school. A narrative study from the family perspective

Abstract: Type 1 diabetes mellitus is one of the most prevalent chronic diseases in childhood, yet it has been little studied in educational research. This research aims to show the school reality of students diagnosed with Diabetes Mellitus type 1, through the story of their families. Qualitative research is carried out, based on the biographical-narrative methodology. Through documentary analysis, interviews and life stories, the concept of inclusive education and its application in the daily life of these students is analysed. The results reflect a school reality far removed from equity, cooperation and justice, essential terms when talking about inclusive education. Based on the he results suggest recognising the importance of family participation in schools to achieve proper control of the disease and foster patients' self-esteem. It also proposes the incorporation of a health worker in schools to alleviate the emotional, economic, and social strain on families. This research gives them the prominence so that their own life stories show their needs and interests.

Keyword: Type 1 Diabetes Mellitus

\section{Introducción}

El sistema educativo ha de servir para que todo el alumnado, independientemente de sus características propias, tenga oportunidades equiparables y de calidad para su correcto desarrollo íntegro (Echeita, 2017). Sin embargo, el fracaso o el éxito educativo no solo radican en las características de los y las estudiantes, sino también en la estructura y en las particularidades de un sistema educativo y social que lo respaldan (Martínez, 2011). Así pues, existen diversos factores como el género, el nivel económico o la salud física y psíquica que obstaculizan la inclusión (UNESCO, 2015). 
A estas variables, hay que añadir las dificultades específicas del alumnado con Diabetes Mellitus tipo 1 (DM1, en adelante) debido a la naturaleza de la enfermedad y la complejidad de su tratamiento. La DM1 es una de las enfermedades graves más frecuentes en la sociedad, pues aumenta su prevalencia e incidencia constantemente (Díaz et al., 2004; Gómez et al., 2009). En España, la incidencia media es de 17.7 por cada 100.000 habitantes, aproximadamente, menores de 14 años; 15.000 niños, niñas y adolescentes afectados (International Diabetes Federation, 2019). Por este motivo, existe una elevada posibilidad de que en las aulas escolares se conviva con alumnos 0 alumnas con dicha enfermedad (Barrio et al., 2020; Gómez et al., 2020).

Debido a esta realidad, en los últimos años, se ha originado un debate acerca de la necesidad de incorporar una figura sanitaria en las escuelas para atender y mejorar la calidad de vida de todos los niños y las niñas, especialmente la de aquellos afectados con enfermedades crónicas, alergias u otras patologías. Por ello, es necesario fomentar la adquisición de capacidades y destrezas en todo el alumnado, incluyendo aquel que, inicialmente, parte de una situación de desventaja, para evitar su posible exclusión social (MEC, 2016); es preciso hacerlo desde una óptica de escuela democrática que incluya a toda la comunidad educativa en su planificación (Puig, 2000).

Para conseguirlo, se demandan transformaciones en el sistema educativo, las cuales provienen, principalmente, de las familias: se necesita conocer sus experiencias. Sus relatos deben ser escuchados, pues de ellos emergerán propuestas de intervención y de modificación en la organización y desarrollo de la educación.

\section{Objetivos}

La finalidad de esta investigación consiste en conocer la realidad que vive el alumnado afectado por DM1 en el ámbito escolar y el significado de la inclusión educativa que emerge del discurso de sus familias. Para ello, nos hemos planteado tres objetivos específicos:

- Conocer el grado de aplicación del principio de inclusión educativa con respecto al alumnado afectado por DM1.

- Explorar la naturaleza y las características de las experiencias y vivencias de inclusión de familias cuyos hijos e hijas padecen DM1.

- Contribuir a la transformación de la situación actual de la relación entre escuela, familias y alumnado afectado por DM1, a través de la mejora de su comprensión.

\section{Método}

El carácter cualitativo de nuestra investigación hace que la palabra, como señala Hernández (2012), sea un elemento fundamental en el procedimiento metodológico empleado para comprender la realidad socioeducativa del alumnado diagnosticado con DM1. De esta manera, las familias son las protagonistas del estudio, pues los testimonios de sus experiencias y vivencias son las que significan la realidad en la que están inmersas.

Partiendo de este carácter cualitativo, nos adentramos en la metodología biográficanarrativa, la cual permite "hablar" a la investigación, pues su carácter "hermenéutico" posibilita dar comprensión y significado a los aspectos afectivos, cognitivos y de acción que conforman las historias de los participantes (Bolívar, et al., 2001). Esto se debe a que, en la investigación, se narran sus propias vivencias, por lo que este método permite al investigador, a los participantes y a los futuros lectores, leer, analizar e interpretar la historia de estas personas (Huchmin y Reyes, 2013). Para ello, acudimos a diferentes 
técnicas metodológicas, de las cuales hemos seleccionado: el análisis documental, la entrevista y el relato de vida.

\section{Instrumentos y técnicas de recogida de datos}

Hemos recurrido a diversas estrategias para construir y reconstruir las historias de los participantes de esta investigación:

Entrevista. La entrevista se ha convertido en una herramienta de considerable eficacia para la obtención de datos en las investigaciones de tipo cualitativo, debido a las interacciones orales que se llevan a cabo (Troncoso-Pantoja y Amaya-Placencia, 2017). Centrándonos en la investigación biográfico-narrativa, Flick (2004) sostiene que la entrevista, con todas sus variantes, es la base de esta metodología.

Dado que precisamos conocer cómo se desarrolla la inclusión del alumnado con DM1 y la implicación que en ella tienen las familias, necesitamos conocer los testimonios de estas. Por ello, recurrimos a la entrevista semiestructurada, pues se define por su carácter flexible en cuanto a la emisión de preguntas. Esto nos permite entrelazar temas, exigiendo al investigador escuchar activamente al entrevistado para adaptarse continuamente a él.

La selección de los participantes debía cumplir unos criterios de inclusión: a) tener un hijo o hija diagnosticado con DM1, b) que este niño o niña estuviera en edad escolar, c) que manifestasen la intención de dialogar activamente con la investigadora y d) que se tuviera un contacto directo entre ambas partes. Una vez seleccionados dichos criterios, nos pusimos en contacto, vía mensajería instantánea con los miembros de la Asociación de Diabetes de Segovia, donde se seleccionaron a dos familias. Se realizaron dos entrevistas de aproximadamente una hora y media de duración, las cuales, se llevaron a cabo en un horario y ambiente cómodo, elegido por los y las participantes. Todo el proceso se grabó con un dispositivo móvil, previo consentimiento informado.

El guion de la entrevista aborda los siguientes temas: momento del debut, vuelta a la realidad familiar, social y escolar y un deseo de futuro. Cabe recordar la necesidad de dejar "hablar" a la investigación, por lo que cada tema a tratar está abordado a través de preguntas abiertas que permiten al entrevistado expresar sus subjetividades, sin sentirse abocado a una respuesta concreta o efímera.

Relatos de vida. "El relato de vida es una entrevista que busca conocer lo social a través de lo individual" (Mallimaci y Giménez, 2006, p. 24). Por ello, se respalda en la experiencia de la persona para profundizar en los valores, las subjetividades y las representaciones de su historia de vida. Este método permite que la persona se apropie y se adueñe de sus vivencias, creando una relación de igualdad entre el propio narrador y el investigador. De este modo, se consigue revivir y reconstruir una historia personal basada en las vivencias, los recuerdos y las valoraciones de sus propios protagonistas (Benadiba, 2015). La transcripción elaborada por el investigador logra que esta historia de vida sea reconstruida, conocida, valorada y escuchada.

Análisis documental. Se puede definir el análisis documental como "una actividad sistemática y planificada que consiste en examinar documentos ya escritos que abarcan una amplia gama de modalidades" (Massot et al., 2004, p. 349). Estos autores afirman que los datos obtenidos a raíz del análisis documental complementarán la información recogida a partir de otras técnicas, lo que posibilita contrastar y validar la información. Partiendo de esta idea, se comenzó con el análisis documental que nos permitió conocer los estudios realizados y hacia dónde debíamos guiar la investigación. Además, los datos extraídos durante el análisis nos ayudaron a contrastar y validar la información obtenida en las entrevistas y en los relatos. 


\section{Análisis de los datos}

Con la elaboración de las entrevistas, explicada en el apartado anterior, obtuvimos la información necesaria para iniciar el análisis, la categorización y la interpretación del relato. Tras el primer análisis, se elaboró un sistema de categorías (tabla 1), el cual permite clasificar la información y catalogarla en unidades, partiendo de un criterio temático o común. Esta categorización permite al investigador adentrarse en el discurso, con el fin de comprenderlo de manera más integral (Mejía, 2011). Estas categorías otorgaron el título inicial a cada capítulo y, posteriormente, esos títulos se fueron reorganizando y renombrando para que los relatos cobraran coherencia y sentido.

\begin{tabular}{ll}
\hline Tabla 1. Sistema de categorías abierto & \\
\hline CATEGORIAS & SUBCATEGORIAS \\
\hline & Educación diabetológica \\
& Trabajo cooperativo \\
& Socialización \\
Medidas inclusoras & Comprensión \\
& Empatía \\
& Amor \\
& Experiencias compartidas \\
\hline & Escaso conocimiento \\
& Escasa formación \\
& Indiferencia \\
& Dificultades sociales \\
& Dificultades económicas \\
Medidas exclusoras & Silencio \\
& Depresión \\
& Incomprensión \\
\hline
\end{tabular}

\section{Resultados}

Este apartado se dividirá en capítulos tal y como se narran los relatos de vida. Cada capítulo contará con transcripciones literales de las entrevistas -presentados en letra cursiva - y los relatos elaborados, indicándose el número de relato o entrevista a la que corresponde (ejemplo: RI: relato primero o El: entrevista primera).

\section{Quiénes somos}

Todos los relatos comenzaron presentando a los protagonistas de la investigación:

La protagonista del primer relato es madre de tres niños, ahora ya, adolescentes. Los dos más pequeños son mellizos y tienen 14 años. Estos jóvenes llevan conviviendo con la diabetes más de 12 años, por lo que tanto ellos como su familia no recuerdan la vida sin diabetes. Debido a la escasa edad con la que fueron diagnosticados con DM1, ha sido su madre la que, desde ese fatídico día, se ha hecho responsable del control de la enfermedad con todo lo que ello implica, -y más, si es por partida doble-. Las patologías de sus hijos, unidas a la exclusión educativa con la que se toparon, no le han permitido desarrollarse a nivel laboral, ni disfrutar a nivel social. Todo ello, la sumió en una gran depresión a la que, con garra y coraje, ha conseguido superar. Aunque la vida no se lo ha puesto nada fácil, ha conseguido tejer alas para aprender a volar, proteger, cuidar y amar a su familia tal y como es. En el relato de su propia historia, esta mamá recuerda esos momentos tristes, duros y dolorosos que tanto la han marcado. A pesar de ello, también consigue desplegar sus alas para recordar, recorrer y sonreír con aquellos buenos momentos que, poco a poco, le ayudaron a tomar las riendas de su vida y ser feliz. (RI)

Los protagonistas del segundo relato son un padre y una madre de dos niños. Su primer hijo convive con la diabetes desde los 5 años -actualmente tiene 8-. El segundo hijo no 
recuerda a su hermano sin diabetes, pues tan solo tiene 5 años y, hoy por hoy, es un niño sano. Este hecho hace que el hermano mayor se pregunte continuamente ¿por qué a mí? Ante esta delicada pregunta, a la que ningún padre o madre querrían tener que contestar, responden ellos: una madre y un padre que desbordan entereza, compromiso y coraje. Una vez más, debido a la escasa edad con la que el niño debutó, son su madre y su padre los que se dividen por las tareas, el control y las responsabilidades diarias de una vida con diabetes. En el relato de sus vidas retroceden y avanzan continuamente en el tiempo para recordar y reconstruir esos momentos que han marcado y definido su camino por la vida. Un camino que ellos han decidido recorrer más unidos que nunca, compartiendo esos momentos, esos logros, esos fracasos y, también, esas lágrimas que, actualmente, deciden no mostrar. Optaron por tejer un escudo protector a base de formación e información que les ayuda a seguir caminando, a superar sus miedos, a vencer sus dudas y, también, a sonreír. (RII)

\section{El debut}

El debut o comienzo de la enfermedad puede constituir, en la mayoría de los casos, una etapa crítica. La persona se enfrenta sin esperarlo y, por tanto, sin estar preparada para ello, a una enfermedad crónica que desajusta toda su vida y que impone un régimen riguroso de tratamiento diario. No solo es necesario tomar o inyectarse el medicamento en un momento del día, sino que debe reajustar toda la actividad diaria a las nuevas demandas que la enfermedad le impone (Sanz, 2020).

Y así sucedió, sin previo aviso, sin permiso y sin quererlo, llegó para quedarse en sus vidas la diabetes. (RI)

El debut fue lo peor (...). Una noche muy mala porque estaba muy deshidratado, no le encontraban las vías para hidratarle ni para ponerle la bomba, controles de sangre cada hora prácticamente, y fue una noche mala, lo pasó muy mal, muy mal, la verdad. (...) Al día siguiente, pues un poco el vacío ese de - juf!, a ver ahora a qué me estoy enfrentando, claro-. (...) Cuando llegó [el padre] a verle por la mañana, pues descorazonado, se echó a llorar el pobre, porque le vio medio crucificado al pobre allí en la cama. Él veía la gravedad de la situación y que no sabíamos a qué nos íbamos a enfrentar, estábamos un poco perdidos, la verdad. Con mucho miedo, (dice el padre) no sabes qué le está pasando, cómo lo vas a afrontar. $Y$ de repente te dicen que te vas a quedar en el hospital al menos quince días, y dices - ijolín!, pues tiene que ser muy grave, muy duro-. (EII)

El niño tenía 18 meses cuando debutó el primero, y luego el segundo a los 23. Entonces, se me vino el mundo abajo. (Al recordarlo se queda sin voz y las lágrimas comienzan a acumularse en sus ojos). (...) Pero bueno, pues poco a poco, te va la gente apoyando, vas buscando soluciones, pero vamos lo peor fue cuando ya tienen que ir al colegio. (EI)

\section{La nueva socialización}

\section{¿Qué cosas han cambiado después del debut?}

Pues todo. (EII)

Y es que ahora, hay algo diferente. Ahora, tú no eres la misma. Ahora, todo cambia.

El ser humano desde que nace es un ser social, destinado a vivir en un mundo social y que, para ello, necesita la ayuda de los demás. Ese ser social, además, se va haciendo poco a poco a través de la interacción con los otros, en un proceso continuo de socialización. (Yubero, 2004, p. 819) 
Pero este proceso de socialización y esa ayuda que antes te brindaba la sociedad, especialmente tu entorno más próximo, se desmorona con la llegada de la diabetes. Ahora, esa socialización también cambia. (RI)

Todo cambia (...). A nivel de amigos, pues tenía un poco de fobia social, porque nosotros una de las cosas que hemos decidido es que cumpleaños va a los justos, a los más amigos. (...) Nos condiciona en que elegimos mucho las ocasiones (...). A la familia también le costó mucho. (EII)

Sus compañeros del cole y del parque y tal, pues sí que les preguntaban y por qué se toma eso, y por qué le pinchas y tal. Y muchos curiosos, o sea curiosidades de a ver qué es lo que le pasa, porque claro no es una enfermedad que salte a la vista, como que está por dentro, entonces como explicárselo a un niño (...). Aunque se lo tenías que explicar casi más a la madre que era más curiosa que el niño.

\section{En el cole}

Los niños protagonistas del primer relato debutaron con apenas año y medio de edad, por lo que, en su primer contacto con el colegio, a los tres años, ya tenían diabetes. La madre pensó que cuando sus hijos se escolarizaran todo cambiaría a mejor. Sin embargo, nada más lejos de la realidad.

Cuando fueron al cole, pues dije, pues ya un respiro - ¡narices, de respiro nada! - (...). Me llamaban cada dos por tres. (EI)

\section{¿Cómo fue ese primer contacto con las profesoras?}

¡Puf...!, pues a ver, (suspira y se emociona al recordar ese momento). Fue muy duro porque las profesoras no se querían hacer responsables de nada y, entonces, nos dijeron que teníamos que dejar teléfonos y que cualquier cosa que ellas notaran en los niños nos iban a llamar. Nos dijeron que teníamos que estar todos los días a la hora del almuerzo para darles el bocadillo y hacerles la prueba, que ellas eran profesoras y no enfermeras. Lo dejaron muy claro (...). Ellas iban solo a dedicarse a su labor que es de maestra, que no son ni médicos ni enfermeras, ni nada, ni siquiera nos los iban a cambiar si se hacían pis (...). Enseñar y enseñar (...), pero vamos, enseñar el temario supongo. En fin, a leer, a escribir, normas de conductas, en fin, esas cosas... Ellas no se dedicaban al tema de la salud, no daban pastillas ni se encargaban de medicinas ni de cosas de médicos (...). Las profesoras dicen: "claro, es que son muchas cosas para estar pendientes" (...). Como diciendo: "nosotros vamos a hacer nuestro trabajo y tú vienes aquí y haces lo que te corresponde a ti”. (EI)

¿Fue similar el recibo del resto de la comunidad educativa?

Hombre, pues al principio yo creo que los veían un poco raros, un poco como bichos raros (nuevamente, las lágrimas comienzan a recorrer sus mejillas). Algo diferentes, porque claro, decían: "¿por qué viene la mamá todos los días a cambiarles, a hacerles el control?". Vamos, ellos no sabían a lo que veníamos porque lo hacíamos fuera. No nos habilitaron una zona, hasta después del primer año, creo que lo hacíamos en el pasillo. (EI)

La escolarización de estos niños supuso un cambio drástico en su calidad de vida y en la de su madre, la única encargada de atender los cuidados de sus hijos incluso durante la jornada escolar.

En fin, yo dejé de hacer vida normal, no te digo más, todo el rato pendiente del teléfono. Ya no es estar pendiente del control, como estoy ahora, sino, si me van a llamar cada vez que le noten al niño raro, voy a estar en el colegio tanto tiempo como las profesoras. (EI) 
La familia protagonista del segundo relato tuvo una situación diferente, pues contaron con una profesora comprometida y con una prestación económica por cuidado de menores afectados por una enfermedad grave.

Fue una situación extraña, porque la profesora fue una interina que solo estaba allí ese año, entonces claro, debutó en marzo, entonces la quedaba hasta junio, entonces se vio con tres meses por delante. Encima sin mucha práctica, no tenía ni idea de la enfermedad, entonces, estaba muy asustada. En cambio, el director llevaba más tiempo y, además, tenía una primita que es diabética, entonces, conocía más el tema. En la reunión, estuvieron un poco asustadas y luego ya se fueron tranquilizando, porque nosotros hemos sido una familia que hemos colaborado, que le hemos dicho: "nada, yo me libero enseguida de trabajar". Entonces yo estaba allí, prácticamente al principio, cada hora y cuarto. Luego tuvimos suerte con la tutora, porque ella se preocupó bastante. O sea, al final, en Infantil que tienen menos desdobles y menos especialidades, entonces tus niños, son tus niños, es mucho más tú problema que en Primaria (...) que es cuando se comenta: "pues yo me quedo al diabético y al asperger y tú te quedas al disléxico y al hiperactivo". (EII)

Esta familia tomó la decisión de acogerse a lo expuesto en el Real Decreto 1148/2011, de 29 de julio, para la aplicación y desarrollo, en el sistema de la Seguridad Social, de la prestación económica por cuidado de menores afectados por cáncer u otra enfermedad grave. En el Preámbulo de este Real Decreto viene recogida la principal finalidad de esta prestación:

Dicha prestación, con naturaleza de subsidio, tiene por objeto compensar la pérdida de ingresos que sufren las personas interesadas al tener que reducir su jornada, con la consiguiente disminución de salarios, ocasionada por la necesidad de cuidar de manera directa, continua y permanente de los hijos o menores a su cargo, durante el tiempo de hospitalización y tratamiento continuado de la enfermedad. El subsidio, por tanto, viene predeterminado por la reducción efectiva de la jornada. (p. 3)

La DM1 está recogida en esta normativa, por lo que uno de los progenitores decidió dejar su trabajo. Sin embargo, a esta prestación no se pueden acoger todas las familias, pues uno de los requisitos es que ambos progenitores estén trabajando.

Quizá, si uno de los dos no trabaja puntualmente, por algún motivo, y quiere volver a hacerlo, ahora se convierta en un imposible reto por las características de la nueva realidad. ¿Nunca podré volver a trabajar? (EI)

\section{¿Quién cuida de la familia?}

Según Corral et al. (2018), la implicación familiar en el control de la enfermedad es imprescindible para lograr un bienestar social y emocional en la persona con diabetes. Asimismo, esta implicación familiar logra que el aprendizaje sobre el manejo de la enfermedad se haga de forma progresiva. Todo ello, permite al paciente madurar cognitiva y emocionalmente y alcanzar, así, la total autonomía.

$Y$ es que, como afirman Dios et al. (2003), "una red social y familiar adecuadas promueven el bienestar emocional y el sentimiento de ser valorado y cuidado por otros, aspectos importantes para una adecuada adaptación a la enfermedad" (p. 171).

Ese sentimiento de ser cuidado por otros, esa comprensión por parte de la sociedad, esa red social a la que tantos autores definen como aspecto clave para conseguir una adecuada adaptación a la enfermedad y que, además, mejora la calidad de vida de los pacientes, también la necesitarían las familias ¿no? Ellas también necesitan ese apoyo social, esa comprensión y esa ayuda a nivel emocional, social, económico... Sin embargo, esas ayudas no existen. Únicamente tienes el amparo médico o el de la 
prestación, ni rastro de políticas de cuidado que brinden una calidad de vida para las familias. (RI)

Me acuerdo de que pedimos ayuda a nuestro médico de cabecera, que fue el que nos puso en contacto que teníamos que ir a un psiquiatra, a un psicólogo, que había un equipo de trabajadores sociales y que, bueno, estuvimos yendo a un montón de sitios y a un montón de cosas. Y bueno, poco a poco vas contando tu experiencia y vas saliendo. (EI)

A ver, lo de la prestación está muy bien, es una gran ayuda, sobre todo, al principio. Entonces, al principio lo veo necesario, pero luego lo que quieres es normalizarlo y tú mismo también, con tu trabajo, seguir con tu vida normal como antes. (Ell)

\section{Soñando con el futuro}

Supongo [narra el padre] que lo que cambiará mucho son los tratamientos. Con las nuevas tecnologías, las bombas irán evolucionado mucho más, los sensores, la toma de decisiones de la diabetes cambiará todo, y luego a nivel educativo, debería de cambiar (...). Y yo creo (cuenta la madre) que el cambio que se va a producir es que va a haber más conciencia con la alimentación (...) y vamos a tener una enfermera en la escuela, y vamos a poder tener un seguimiento controlado, pero con menos marcaje, porque al final, el padre cuando aparece por ahí es un marcaje que, aunque el niño lo lleve bien, en fin... Y tendremos mejor tecnología. Y luego habrá más padres formados que los que hay ahora y que podremos ayudarles también y que estamos dispuestos, ¿verdad? (sus ojos están iluminados y ambos sonríen con un gesto cómplice), porque las asociaciones también hacen una labor muy buena, y que sigan por ahí. (EII)

Espero que todo esto haya cambiado bastante, espero. Porque, de hecho, se está pensando en poner una enfermera en los colegios. Entonces, esto va a hacer que la tranquilidad de las mamás cuando se vayan a sus casas y dejen a los niños en el cole, pues eso se va a notar (...). Para la salud de los niños y para la salud del entorno, porque es que ganamos todos, los padres, los niños y el entorno; la tranquilidad. (EI)

\section{Discusión y conclusiones}

En primer lugar, queremos rescatar la idea de incorporar una figura sanitaria en la escuela como medida inclusora. En este punto García (2019) señala que, a partir de su presencia y labor en las escuelas e institutos, los adolescentes con DM1 suelen acceder a hábitos más saludables, mejorando su calidad de vida. Por su parte, Bodas et al. (2008), presentan amplias coherencias con los anhelos que exponen los participantes de este estudio: los niños y niñas solicitan en casi un $45 \%$ de los casos la presencia de una enfermera en su centro educativo.

En síntesis, la enfermera escolar sería fundamental para desarrollar una atención correcta y coherente con las necesidades del alumnado con enfermedades crónicas, por ello, es totalmente necesaria en la organización de las escuelas (Ruiz et al., 2015).

En segundo lugar, debemos destacar el desconocimiento generalizado que existe sobre la DM1. Este desconocimiento queda reflejado en los estudios de Bodas et al. (2008), Martínez et al. (2012) y Rodríguez (2019). Asimismo, en los resultados de nuestra investigación hemos podido apreciar este desconocimiento generalizado cuando los participantes nos relatan, basándose en sus experiencias, el desconocimiento que ellos mismos tienen ante el debut de la enfermedad.

Por todo lo dicho anteriormente, estos trabajos ofrecen una realidad educativa bastante alejada de la educación inclusiva. La escuela no afronta ni se adapta a la nueva realidad de este alumnado y de sus familias. La escuela mira hacia otro lado, abogando a que el profesorado únicamente se dedica a enseñar, pero ¿qué es enseñar? 
Los estudios que hablan del alumnado diagnosticado con DM1 en Educación Infantil o sobre el alumnado que, debido a su temprana edad, aún no sabe autocontrolar su enfermedad (menores de 12 años, aproximadamente) son escasos (Wagner y James, 2006; de Cássia et al., 2017) y suelen centrarse en las características médicas de la enfermedad más que en su efectivo tratamiento en las aulas. Además, no se han publicado estudios biográfico-narrativos centrados en las familias de niños y niñas con DM1 en Hispanoamérica, por lo que es difícil discutir la investigación que se expone en estas líneas.

Declaramos que las vivencias educativas que enfrentan las familias y el alumnado diagnosticado con DM1 exponen una realidad que refleja la injusticia social que conlleva tener una enfermedad. Una realidad que necesita ser escuchada y comprendida para ser transformada.

Actualmente, la inclusión sigue siendo un reto para nuestro sistema educativo, pero también un proceso que pretende ayudar a transformar las escuelas para que todo el alumnado, sin exclusión alguna, tengan igualdad de oportunidades para el desarrollo de su personalidad (Echeita, 2017). Por tanto, hablar de inclusión no es solo integrar al alumnado en un centro ordinario, sino que supone una transformación de todo el contexto educativo.

La inclusión educativa es necesaria para garantizar la equidad, y por ello es obligatorio que prime la atención individual de cada alumno y alumna. El alumnado diagnosticado con DM1 puede estar integrado en un centro ordinario, pero mientras ese contexto educativo no atienda sus necesidades e intereses individuales, no comprenda ni empatice con su situación de vulnerabilidad, no garantice su seguridad, no coopere con las familias; en definitiva, mientras el contexto educativo siga mirando hacia otro lado, estigmatizando con sus filas de "los especiales", homogeneizando, compitiendo y coartando la identidad del alumnado, no podremos hablar de inclusión educativa.

Los trabajos de Bodas et al. (2008); Martínez et al. (2012) y Ruiz et al. (2015) muestran las barreras exclusoras con las que diariamente se topa este alumnado, como son: la escasez de formación y la desinformación sobre esta enfermedad, la falta de compromiso y empatía por parte de la comunidad educativa, la falta de recursos humanos y materiales o la nula cooperación entre profesionales.

Centrándonos en esa escasez de formación en el ámbito educativo, Rodríguez (2019) y García (2019) manifiestan la necesidad de formar y apoyar a la comunidad educativa para mejorar la calidad de vida del alumnado diagnosticado con DM1, pues este simple hecho les aportaría seguridad y tranquilidad en su día a día. Martínez et al. (2012) va un paso más allá en su investigación y se propone formar a la comunidad educativa a través de una intervención educativa. Sus resultados evidenciaron que el $97 \%$ de los participantes valoró como "buena" o "muy buena" dicha intervención.

En los relatos construidos para nuestra investigación, además de reafirmarse la escasez de formación e información, se habla incluso de fobia social, miedos y estigmas. Una vez más, se tiende a comparar, a encasillar o etiquetar a aquellos alumnos que son iguales y a aquellos que son diferentes.

Esta investigación nos mostró la posibilidad de conocer los intereses y las reivindicaciones de familias cuyos hijos e hijas han sido diagnosticados con DM1. Ahora sabemos que, en este contexto, el grado de aplicación del principio de inclusión educativa con respecto a este alumnado aún está lejos de alcanzar la meta.

Este estudio refleja la necesidad que tiene el ser humano de socializar, de ser escuchado, comprendido y querido por los demás. Desde ese duro comienzo con el debut de la enfermedad y su rotunda transformación de la realidad, surgen las primeras 
demandas socializadoras. Las familias necesitan ayuda, requieren empatía, comprensión, tolerancia y apoyo de los demás. Tras la larga estancia hospitalaria que requiere la enfermedad, deben volver a su nueva realidad. Esta realidad, de ahora en adelante, estará marcada por la diabetes. Sus relaciones sociales se ven involucradas por la desinformación y el desconocimiento, por el miedo, por la incomprensión y por las incómodas preguntas que, en numerosas ocasiones, agotan. También se ven afectados los aspectos económicos al tener que dejar de trabajar, uno de los dos progenitores, para poder atender las nuevas necesidades de su hijo o hija, ahora, con una enfermedad. Esto se debe a que no existen ni recursos sociales, ni recursos educativos, ni recursos médicos que posibiliten una correcta adaptación de estos niños a su nueva realidad.

A nivel educativo, hemos comprobado que los niños y las niñas diagnosticados con DM1 están escolarizados en centros ordinarios, por lo que en ese sentido puede predicarse la integración, no la inclusión de este alumnado. Las familias y el centro educativo deben cooperar, pero esto no siempre sucede. Desde las escuelas se requiere el continuo apoyo de las familias, pero no para cooperar, sino para deshacerse de su obligación, para mirar hacia otro lado y creer que no pasa nada. Pero claro que pasa algo, y algo muy serio: hay un colectivo de niños y niñas que os necesitan, que quieren mantener su propia identidad y no a base de ser el "especial" o a base de autoexcluirse, sino a base de comprensión, tolerancia, respeto y, ¿por qué no?, inclusión.

Llegados a este punto, somos conscientes de que la perspectiva inclusiva nos ha permitido conocer los intereses y las necesidades del alumnado afectado por DM1. Hemos comprobado que la inclusión de este alumnado en la escuela presenta severas dificultades debido a la naturaleza de la enfermedad y al complicado tratamiento que esta conlleva. De ahí, que se precise una coordinación entre las administraciones públicas de educación, sanidad y asuntos sociales, puesto que esto facilitaría el proceso de inclusión, garantizaría la seguridad y la viabilidad del tratamiento y ofrecería esa igualdad de oportunidades (Bodas et al. 2008).

A lo largo de la investigación hemos comprendido que la implicación familiar es imprescindible para fomentar la autoestima, la confianza, la seguridad y, además, para mejorar el autocontrol de la enfermedad. En esta misma línea, se hace indispensable la formación y el apoyo a la comunidad educativa para contribuir a la transformación del proceso de inclusión.

Por último, debemos comprender que, para contribuir con esta transformación social, el trabajo cooperativo entre familia-escuela-equipo sanitario es imprescindible. También, debemos concientizarnos de que esta transformación requiere de una autotransformación. Es necesario que estemos dispuestos a desaprender para seguir aprendiendo, a coaprender, a tolerar, a empatizar y a respetar que cada uno elija ser igual o diferente en lo que quiera, hasta donde quiera y para lo que quiera.

\section{Referencias}

Barrio, R, Méndez, P y Rodergas, J (2020). El niño, la diabetes y la escuela. Fundación para la Diabetes. https://cutt.ly/XIs9Abl

Benadiba, L (2015). Historia oral: reconstruir historias únicas desde la diversidad. Revista Confluências Culturais, 4(2), 90-99. https://cutt.ly/alk9VDa

Bodas, P, Marín, MC, Amillategui, B y Arana, R (2008). Diabetes en la escuela. Percepciones de los niños y adolescentes con diabetes mellitus tipo. Avances en diabetología, 24(1), 51-55. https://cutt.ly/sls8qFG
Bolívar, A, Segovia, D y Fernández, M (2001). La investigación biográfico-narrativa en educación. Enfoque y metodología. Muralla.

Corral, MG, Sevilla, M, Gallardo, I, Guillén, J y Sánchez-Monge, M (2018). La importancia de la familia en la diabetes. CuídatePlus. https://cutt.ly/yllcbva

Dios (de), C, Avedillo, C, Palao, A, Ortiz, A y Agud, JL (2003). Factores familiares y sociales asociados al bienestar emocional en adolescentes diabéticos. The European journal of psychiatry, 17(3), 171-182. https://cutt.ly/9ls81wz 
Echeita, G (2017). Educación inclusiva. Sonrisas y lágrimas. Aula Abierta, (46), 17-24. https://doi.org/10.17811/rifie.46.2.2017.17-24

Flick, U (2004). Introducción a la investigación cualitativa. Morata.

García, ME (2019). Gamificación aplicada a la Educación para la Salud de adolescentes con el diagnóstico de Diabetes Mellitus tipo 1 [tesis doctoral, Universitat de València]. https://cutt.ly/dls5KBK

Gómez, G, Carollo, M, Abelairas, C, Sánchez, L, García, FM y Rodríguez, A (2020). Escolares con enfermedades crónicas, ¿qué les preocupa a sus profesores? Anales de Pediatría, 93(6), 374379. https://doi.org/10.1016/j.anpedi.2020.02.004

Gómez, M, Gómez, JA, Ramírez, J, Díez, T y García, E (2009). Necesidades del niño escolarizado con diabetes mellitus. Visión de padres y profesores. Anales de Pediatría, 70(1), 45-52. https://doi.org/10.1016/j.anpedi.2008.07.004

Hernández, I (2012). Investigación cualitativa: una metodología en marcha sobre el hecho social. Rastros Rostros, 14(27), 57-68.

Huchim, D y Reyes, R (2013). La investigación biográfico-narrativa, una alternativa para el estudio de los docentes. Actualidades investigativas en Educación, 13(3), 392-419. https://cutt.ly/3IdqoMr

International Diabetes Federation (2019). Diabetes Atlas. http://www.diabetesatlas.org/

Mallimaci, F y Giménez, V (2006). Historia de vida y métodos biográficos. En I. Vasilachis (coord.), Estrategias de investigación cualitativa (pp. 175-212). Gedisa.

Martínez, A, García, R, Arranz, E, García, MC y Comellas, M (2012). Tengo diabetes. ¿Quién cuida de mí en la escuela? Revista Iberoamericana de Enfermería Comunitaria, 5(2), 32-34. https://cutt.ly/MIdrXEE

Martínez, B (2011). Luces y sombras de las medidas de atención a la diversidad en el camino de la inclusión educativa. Revista Interuniversitaria de formación del profesorado, 70(25), 165-183. https://cutt.ly/Jldta3a

Massot, I, Dorio, I y Sabariego, M (2004). Estrategias de recogida y análisis de la información. En R. Bisquerra (coord.), Metodología de la Investigación Educativa (pp. 329-366). La Muralla.

Mejía, J (2011). Problemas centrales del análisis de datos cualitativos. Revista latinoamericana de Metodología de la Investigación Social, 1, 47-60. https://cutt.ly/vlk7RRr

Ministerio de Educación, Cultura y Deporte. (2016). PISA 2015. Programa para la Evaluación Internacional de los Alumnos. Informe Español. https://cutt.ly/EIduEEH

Ministerio de Trabajo e Inmigración. (2011, 30 de julio). Real Decreto 1148. Para la aplicación y desarrollo, en el sistema de la Seguridad Social, de la prestación económica por cuidado de menores afectados por cáncer y otra enfermedad grave. Boletín Oficial del Estado. 182. https://cutt.ly/lldoOPK

Prieto, MA (2019). Impacto de la escuela de pacientes en la autogestión de las enfermedades crónicas [Tesis doctoral, Universidad de Sevilla]. Depósito de Investigación Universidad de Sevilla. https://hdl.handle.net/11441/85391
Puig, JM (2000). ¿Cómo hacer escuelas democráticas? Educação e Pesquisa, 26(2), 55-59. https://doi.org/10.1590/S151797022000000200005

Ruiz, R, Ferrer, JL y Postigo, S (2015). La enfermera escolar y la enfermera de Atención Primaria. A propósito de la atención a la diabetes en la escuela en Extremadura. Revista Rol de Enfermería, 38(9), 8-14.

Sanz, O (2020). Debut y fases de adaptación. Fundación para la Diabetes. https://cutt.ly/Gllt7D6

Troncoso-Pantoja, C y Amaya-Placencia, A (2017). Entrevista: guía práctica para la recolección de datos cualitativos en investigación de salud. Revista de la Facultad de Medicina, 65(2), 329-332. http://dx.doi.org/10.15446/revfacmed.v65n2.60235

UNESCO. (2015). Replantear la educación ¿Hacia un bien común mundial?. UNESCO.

Yubero, S (2004). Socialización y aprendizaje social. En I Fernández, S Ubillos, EM Zubieta y D. Páez (coords.), Psicología social, cultura y educación (pp. 819-844). Pearson. 